\title{
Medindo a qualidade de geradores de números aleatórios
}

\author{
Daniel Chicayban Bastos ${ }^{1}$ \\ Luis Antonio Brasil Kowada ${ }^{1}$ \\ ${ }^{1}$ Universidade Federal Fluminense \\ Instituto de Computação \\ Av. Gal. Milton Tavares de Souza \\ São Domingos, Niterói, RJ, 24210-310
}

\begin{abstract}
Resumo. Conseguir geradores de números aleatórios satisfatórios não é uma tarefa simples. A maioria falha em diferentes testes estatísticos, por possuírem padrões que são revelados nestes testes. Neste trabalho, apresentamos algumas dificuldades teóricas para a geração de números aleatórios, o estado da arte em testes estatísticos e discutimos propriedades e problemas em aberto para comparar e classificar os diferentes geradores.
\end{abstract}

\begin{abstract}
Designing adequate procedures to produce random numbers is no simple task. Most generators fail statistical tests, revealing patterns in their output. We present some theoretical difficulties in the generation of random numbers, the state-of-the-art in statistical tests and discuss properties and open problems to compare and classify generators.
\end{abstract}

\section{Introdução}

A importância de se distinguir geradores satisfatórios de geradores insatisfatórios decorre de suas aplicações. Dentre as aplicações mais dependentes de boa geração de números aleatórios está a criptografia. A má qualidade de um gerador de números aleatórios pode implicar a insegurança da criptografia.

Infelizmente casos desastrosos em função de geradores de números aleatórios acontecem. A tragédia mais antiga talvez seja RANDU, usado no System/370 da IBM. O gerador é popularmente conhecido como o pior gerador já proposto. "[Its] very name $R A N D U$ is enough to bring dismay into the eyes and stomachs of many computer cientists! [...] [It] fails most three dimensional criteria for randomness, and it should never have been used." [Knuth 1997, capítulo 3, seção 3.3.4, "the spectral test", página 105]

Em 1996, ocorreu o famoso caso de insegurança do navegador Netscape ao escolher uma chave para estabelecer o canal seguro inicial de comunicação com um servidor web [Goldberg and Wagner 1996]. O problema com a geração de números aleatórios do navegador não era com o gerador per se, mas com a informação inicial usada para alimentar o gerador, isto é, a semente do gerador, chamada de seed. A Netscape Communications usou como seed a data atual do momento da geração das chaves com exatidão

Trabalho parcialmente apoiado pelo projeto SHCDCiber. Autores listados em ordem alfabética: veja declaração da AMS em https://goo.gl/rzBAq9. 
máxima de um segundo, além do $\mathrm{PID}^{1}$ e do PPID do processo do navegador. O momento da geração das chaves é obtido trivialmente por um adversário com capacidade de observar a comunicação entre o navegador e o servidor web. O P ID e PP ID são trivialmente obtidos por um usuário local ao computador onde se executa o navegador e, mesmo não sendo um usuário local, o universo de P ID e PP ID de processos de sistemas operacionais não é grande o suficiente para não restringir o universo de chaves. A semente propriamente era computada pelo algoritmo MD5 [Rivest 1992], que em 1996 ainda não tinha tido sua segurança comprometida, mas a entrada para o procedimento de Rivest, que computava o seed era restrita o suficiente para inutilizar a segurança oferecida pelo MD5 e pelo esquema RSA adotados pela Netscape Communications. A empresa anunciava uma segurança de 128 bits, que na verdade era de 47 bits em virtude da fraqueza de seu procedimento de geração de chaves [Goldberg and Wagner 1996, seção "attacks on netscape"].

Em 2003, Taiwan iniciou um projeto oferecendo aos taiwaneses um cartão inteligente que os permitiam se autenticar com o governo, declarar imposto de renda e regularizar a documentação de veículos, tudo pela Internet. Chaves RSA eram geradas pelos cartões, assinadas pelo governo e armazenadas em um repositório na Internet. Alguns cartões, entretanto, possuíam geradores gravemente defeituosos. Em 2013, foram fatoradas 184 chaves RSA distintas de um universo de mais de dois milhões de chaves RSA de 1024 bits, sendo obtida assim a chave privada de cada uma dessas 184 chaves públicas [Bernstein et al. 2013].

Em 2012, uma análise ampla da qualidade de geração de chaves públicas usadas na Internet pelos protocolos TLS e SSH foi feita [Heninger et al. 2012]. Varreu-se todo o espaço de endereços IPv4 procurando-se por servidores TLS e SSH, permitindonos uma visão macroscópica do universo de chaves existente na Internet. Coletou-se 5,8 milhões de certificados TLS distintos e 6,2 milhões de chaves SSH distintas, oriundos de 10,2 milhões de computadores. Descobriu-se que pelo menos 5,57\% dos servidores TLS e $9,60 \%$ dos servidores SSH usavam uma chave comum com pelo menos um outro servidor. No caso do protocolo TLS, pelo menos 5,23\% dos computadores usavam chaves escolhidas pelo fabricante do serviço e nunca teriam sido alteradas pelo usuário. Outros $0,34 \%$ teriam escolhido chaves iguais a outros computadores devido a geradores de números aleatórios defeituosos. Eles conseguiram obter a chave privada de 0,50\% dos servidores TLS e de $0,03 \%$ dos servidores SSH, explorando o fato de que algumas chaves públicas compartilhavam fatores comuns devido à má geração de números aleatórios. Conseguiu-se também recuperar 1,03\% das chaves privadas de algoritmos de assinatura digital de servidores $\mathrm{SSH}$, também devido a defeitos na geração de números aleatórios usada por esses servidores. Em números absolutos, aproximadamente 64 mil chaves privadas de servidores TLS foram obtidas e 108 mil chaves privadas de servidores SSH, todas devido a defeito na geração de números aleatórios [Heninger et al. 2012, seção 1, "introduction and roadmap"].

Outro aspecto de segurança é a deterioração da qualidade de serviços. Geradores de números aleatórios também são alvos de ataques nesse sentido. Suponha que um

\footnotetext{
${ }^{1}$ P ID significa process identifier e P P ID é o P ID do processo pai em sistemas UNIX. No kernel Linux, em sua versão 2.5.68, todo P ID é um número natural entre 1 e 32767 em sistemas 32-bits. Em sistemas 64-bits o valor pode chegar a $2^{22}$, aproximadamente 4.2 milhões [Linux Programmer's Manual 2017].
} 
certo serviço popular utilize Quicksort [Hoare 1962] aleatório como parte de seu serviço e utilize um gerador de números aleatórios de má qualidade de forma que consigamos prevê-lo. Se o sorteio do pivot é de fato aleatório, o método opera com tempo médio de $\Theta(n \lg n)$, sendo $n$ o tamanho do conjunto de dados a ser ordenado. No pior caso, o custo sobe para $\Theta\left(n^{2}\right)$. Sendo assim, podemos projetar um ataque ao serviço, gerando conjuntos de dados com a exata composição que force Quicksort a operar sempre em seu pior caso. Reduzimos efetivamente a qualidade do serviço.

Outras áreas importantes de aplicação de geradores de números aleatórios incluem simulação de modelos, amostragem, análise numérica, recreação. Em simulações, temos previsão do tempo e fenômenos físicos como a colisão de partículas atômicas. Em amostragem, uma seleção aleatória ilustra o que constitui comportamento típico [Knuth 1997, capítulo 3, seção 3.1, item "b", página 2]. Em análise numérica, temos a elegância do algoritmo rô de John M. Pollard, que usa números pseudo-aleatórios em uma forma de buscar um fator não-trivial de um número composto [Pollard 1975]. Em recreação incluímos o lançamento de dados, típico em jogos de tabuleiro, o sorteio de cartas de baralho, roletas de cassino et cetera.

\section{Terminologia}

A literatura distingue procedimentos aritméticos de procedimentos físicos, como variações de correntes elétricas ou emissão de partículas subatômicas, usados para a geração dessas sequências aleatórias. Quando o procedimento é aritmético, o gerador é chamado de pseudo-aleatório e é referenciado pela sigla PRNG, do inglês pseudorandom number generator. Quando o procedimento é físico, é comum se usar a sigla RNG, do inglês random number generator. Quando o procedimento é um PRNG considerado seguro para aplicações criptográficas, ele é referenciado pela sigla CSPRNG, do inglês cryptographically secure pseudo-random number generator.

\section{Verdadeira aleatoriedade}

O conceito de verdadeira aleatoriedade oferece vários desafios. A teoria da probabilidade, por exemplo, evita a dificuldade de definir o que se quer dizer por uma sequência "aleatória". A teoria expressa suas noções quantitativamente - quanto de probabilidade se deve atribuir a eventos. O objetivo é medir e não dar significado [Knuth 1997, capítulo 3, seção 3.5, página 149].

Consideremos, por exemplo, como aleatória uma sequência pelo menos uniforme de forma que possamos gerar chaves criptográficas sem qualquer viés; de forma que tenhamos um universo equiprovável de chaves. Por clareza, considere uma sequência $P$ com 32 bits de estado, sendo capaz de gerar $2^{32}$ elementos, aparecendo cada um uma única vez na sequência. $P$ gera elementos uniformemente distribuídos, mas sua natureza "aleatória" é questionável: se lançamos uma moeda uniforme de período fixo de tamanho 2 e obtemos um de seus lados, seríamos obrigados a esperar que obteríamos seu outro lado no próximo lançamento, o que é absurdo. O que ocorre nesse experimento é que o período dessa moeda é 2, muito curto. A capacidade da moeda de se mostrar aparentemente aleatória é esgotada já no primeiro lançamento. Similarmente, nenhum elemento de $P$ poderá aparecer mais de uma vez, fracassando eventualmente no teste estatístico baseado no problema do aniversário - que diz efetivamente que se coletarmos 
365 números de uma sequência verdadeiramente aleatória, encontraremos uma repetição qualquer, com probabilidade maior que 50\%, coletando-se apenas 23 números. Sendo assim, uma sequência de 32 bits uniforme fracassará em algum teste estatístico à medida que seus elementos sejam consumidos.

Como possível contra-argumento, consideremos uma sequência $Q$ uniforme de 32 bits de estado, mas cuja imagem possua apenas $2^{16}$ elementos. Assim, cada elemento de $Q$ aparecerá $2^{16}$ vezes, o que poderia nos sugerir que as dificuldades com o problema do aniversário pudessem ser eliminadas. Podemos visualizar $Q$ como uma coleção de caixas, cada uma contendo $2^{16}$ números. Por exemplo, $Q$ possui uma caixa contendo $2^{16}$ números sete. Na primeira vez que $Q$ produza um número sete, a caixa referente ao sete perde um deles e passa a ter $2^{16}-1$ setes. Eventualmente $Q$ perderá todos os setes e nenhum outro sete será produzido até o fim do período de $Q$. Quando $Q$ já tiver produzido todos exceto seu último elemento, só haverá uma possível resposta para o último elemento de $Q$, o que é nada aleatório, implicando que todos os geradores uniformes eventualmente fracassarão em algum teste estatístico [O’Neill 2014, seção 3.1, página 15].

\section{Análise de evidências pelo teste de hipótese}

Suponha que afirmemos que uma certa sequência seja aleatória. Estatísticos chamam tal suposição de hipótese nula, $H_{0}$. A inferência estatística nos permite aplicar testes de forma que possamos aceitar $H_{0}$, rejeitar $H_{0}$ ou considerar o teste inconclusivo. Ao aceitarmos ou rejeitarmos $H_{0}$, podemos fazê-lo erroneamente. Por exemplo, a sequência pode ser de fato aleatória e determinarmos incorretamente que ela não o seja, o que podemos chamar de erro 1. Por outro lado, a sequência pode não ser aleatória e determinarmos que seja, o que podemos chamar de erro 2. A nós o erro 2 é mais grave que o erro 1: quando incorremos no erro 2, obtemos a falsa segurança de que temos um gerador satisfatório quando de fato não temos, enquanto no erro 1 ainda estamos em segurança, apesar de não possuir os fatos.

Existe uma probabilidade de incorrermos em qualquer desses erros. A probabilidade do erro 1 é chamada de nível de significância do teste e $\alpha$ é o símbolo usualmente atribuído a ela, ou seja, $\alpha$ é a probabilidade de que o teste indique que a sequência não seja aleatória quando ela de fato é.

Similarmente, atribui-se a $\beta$ a probabilidade do erro 2. Diferente de $\alpha, \beta$ pode assumir diversos valores porque existem infinitas formas em que uma sequência possa apresentar certa estrutura que a qualifique como não-aleatória. Cada uma dessas formas gera um valor $\beta$ diferente.

As probabilidades $\alpha$ e $\beta$ relacionam-se entre em si bem como com o tamanho $n$ da amostra da sequência sob escrutínio. Usualmente escolhe-se um valor para $\alpha$, um tamanho $n$ para a amostra da sequência e define-se um valor crítico relativo a uma regra de decisão para aceitar ou rejeitar $H_{0}$.

Dado um teste estatístico, a teoria do teste de hipótese nos permite computar uma medida, chamada de $p$-valor, que resume a força da evidência contra a hipótese $H_{0}$. Esse procedimento é análogo a um argumento por contradição: a hipótese $H_{0}$ é aceita se sua negação é improvável. 


\section{O estado da arte em testes estatísticos}

A partir do teste de hipótese, pode-se conceber um sem-número de testes estatísticos pelos quais todo gerador satisfatório deve passar. Entretanto, não existe conjunto finito de testes estatísticos que seja suficiente ao propósito de testar geradores. Se uma sequência se apresenta como aleatória sob a ótica dos testes que aplicamos, nunca poderemos ter certeza de que a sequência é de fato aleatória aos nossos propósitos, mas quanto mais testes aplicamos e quanto mais perspicazes forem, mais confiantes estaremos e assim a sequência é presumidamente aleatória até que se prove o contrário [Knuth 1997, capítulo 3, "statistical tests", página 41].

A primeira bateria de testes sobre a qualidade da geração de números aleatórios começou em 1969 [Knuth 1997, seção 3.3, "statistical tests"]. Em 1996 apareceu o pacote DIEHARD [Marsaglia 1996], em virtude da insuficiência dos testes de Knuth. Em 2000 veio a publicação do NIST SP 800-22 [Bassham III et al. 2010]. Em 2004, DieHarder [Brown 2004], em virtude da insufiência do DIEHARD. Em paralelo, em 2007 foi publicado o pacote TestU01 [L'Ecuyer and Simard 2007, L'Ecuyer and Simard 2005], uma biblioteca $\mathrm{C}$ contendo testes estatísticos para relatar sobre a qualidade de geradores de números aleatórios:

[...] empirical testing of RNGs is very important, and yet no comprehensive, flexible, state-of-the-art software is available for that, aside from the one we are now introducing. The aim of the TestU01 library is to provide a general and extensive set of software tools for statistical testing of RNGs. It implements a larger variety of tests than any other available competing library we know. [...] Test $U 01$ was developed and refined during the past 15 years and beta versions have been available over the Internet for a few years already. It will be maintained and updated on a regular basis in the future.

Sem publicação formal, concorrem com o TestU01 o gjrand [Johnson 2014] bem como o PractRand [Doty-Humphrey 2014].

\section{Comparando e classificando geradores}

Dado que dois geradores $A$ e $B$ passem em determinada bateria de testes, é natural que perguntemos qual dos dois se deve usar. Se ambos obtêm sucesso contra o estado da arte em testes, então precisamos de uma métrica de comparação.

Já sabemos que um gerador uniforme de $b$ bits de estado interno eventualmente fracassará em algum teste estatístico. Devido a seu período fixo, todo gerador é esgotável por algum teste que o leve ao extremo por consumi-lo por completo. Dado um teste estatístico específico, é possível determinar quantos bits serão consumidos do gerador e a partir daí computar quantos números serão necessários para que o gerador fracasse no teste. Isso nos permite colocar limites que mesmo um gerador ideal com as características que desejamos, como uniformidade, precisa respeitar. Por exemplo, mesmo um gerador ideal levado ao extremo fracassará na pequena bateria de testes, SmallCrush [L'Ecuyer and Simard 2007], se o tamanho de seu estado interno for menor que 32 bits; fracassará na bateria média, Crush, se $b<35$; fracassará na BigCrush se $b<36$ [O’Neill 2014, seção 3.2, página 18]. Isso significa que um gerador de $b=32$ bits não tem como passar pela bateria BigCrush, mas tem seu mérito se puder passar pela SmallCrush com um mínimo de estado. 
Se um gerador fracassa em algum teste estatístico, dar-lhe mais bits de estado pode torná-lo bem-sucedido. O converso também é verdadeiro: se um gerador passa num teste, reduzir seu estado interno pode fazê-lo não passar. Por exemplo, considerando apenas o tamanho do estado interno, se um gerador $A$ passa num teste estatístico usando $b$ bits de estado e $B$ precisa de $b+k$ bits para fazer o mesmo, então $A$ é superior a $B$ porque possui $k$ bits de folga relativo a $B$ [O’Neill 2014, seção 3.2, página 19].

Temos aqui um problema em aberto. Suponha que um gerador $X$ precise de 63 bits de estado para passar num certo teste $T$ e fracasse em $T$ se for reduzido para 62 bits de estado. Isso significa que 63 bits é o mínimo necessário para $X$ obter sucesso em $T$. Isso sugere que provavelmente se $T$ coletar amostra de maior tamanho, $X$ não passará em $T$. Em contrapartida, se $Y$ passa em $T$ com uma folga de 24 bits, podemos esperar que $Y$ possua maior margem de tamanho de amostra que $T$ possa coletar. É verdadeiro que $b$ bits de folga representem a mesma coisa para geradores diferentes? [O'Neill 2014, seção 8, "future work", página 48] Se não for, seria possível definir uma métrica relativa à folga que não seja injusta a qualquer dos geradores sob comparação?

À medida que aumentamos o estado interno de um gerador, ele tende a fracassar em menos testes. A partir do número de fracassos em função do tamanho do estado interno, podemos construir uma curva contínua e então computar a velocidade de declínio dessa curva em algum ponto em que a derivada da curva exista. Sendo assim, podemos determinar que um gerador $X$ reduza seu número de fracassos a zero mais rápido que um gerador $Y$. Seria essa mais veloz redução de fracasso evidência de uma característica particular do gerador? [O’Neill 2014, seção 8, "future work", página 48] Seria uma característica de $X$ necessariamente superior a $Y$ ? Seria possível definir uma métrica útil a partir dessa velocidade de redução de fracasso em função do aumento do tamanho do estado interno?

Essas são perguntas que estão atualmente sob nossa consideração.

\section{Geradores criptograficamente seguros}

Não basta testar estatisticamente um gerador e declará-lo adequado à criptografia. "Passing [such tests] would certainly be necessary for cryptographic security, but it is not sufficient.” [Stinson 2006, capítulo 8, seção 8.1, página 324]

Para analisar a segurança de um CSPRNG, podemos recorrer a algumas definições a respeito de segurança de sistemas criptográficos [Stinson 2006, capítulo 2, seções 2.1, 2.3 , páginas 45,50 , capítulo 8 , seção 8.2 , página 327] e fazer um paralelo com geradores de números aleatórios.

Definição 7.1 (computacionalmente seguro). Um PRNG é chamado de computacionalmente seguro se o melhor algoritmo para violar sua segurança requer pelo menos $N$ operações, sendo $N$ algum natural específico grande.

Considere um CSPRNG $P$ de período $2^{100}$. Podemos prever $P$ por completo coletando $2^{100}$ amostras de seu ciclo, o que é óbvio, visto que esgotamos seu período. Sendo essa a melhor estratégia para se violar $P$, então $P$ seria computacionalmente seguro.

Definição 7.2 (comprovadamente seguro). Um PRNG é chamado de comprovadamente seguro se violar sua segurança significa necessariamente solucionar em tempo polinomial um problema considerado difícil. 
A situação aqui é análoga a provar a NP-completude de algum problema, pois reduzimos a dificuldade de ataque de um PRNG à dificuldade de se solucionar um problema considerado difícil. Por exemplo, o criptossistema Rabin é equivalente ao problema da fatoração [Stinson 2006, capítulo 5, seção 5.8, página 211], que é considerado difícil dado o modelo de computação clássico. Como consequência, podemos conceber um PRNG baseado no criptossistema Rabin e declará-lo comprovadamente seguro.

Definição 7.3 (segredo perfeito). Um PRNG possui segredo perfeito se não se pode obter qualquer informação sobre seu estado interno a partir de seu output.

Um gerador verdadeiramente aleatório satisfaria a definição de segredo perfeito, visto que por definição um gerador verdadeiramente aleatório não relaciona a saída com sua entrada.

Em geral não é fácil provar que certo PRNG satisfaça essas definições, como não é fácil provar que criptossistemas as satisfaçam [Stinson 2006, capítulo 2, seção 2.1, página 45]. Usualmente a segurança de um criptossistema é dada relativa a uma estratégia de ataque específica, como força bruta e o mesmo pode ser feito para a análise de um PRNG. É claro que segurança contra uma certa estratégia de ataque não garante o sistema contra outros tipos de ataques [Stinson 2006, capítulo 2, seção 2.1, página 45], o que também se aplica a um PRNG.

Analisar a segurança de um PRNG portanto requer análise de especialistas. Como consequência, recomendações de CSPRNG decorrem do consenso da comunidade. Por exemplo, atualmente um CSPRNG popular é o ChaCha20 [Bernstein 2008], o que se evidencia pelo fato de ter tomado o lugar do RC4 no sistema OpenBSD em sua versão 5.4, substituindo o RC4 também no NetBSD em sua versão 7.0 e substituindo SHA-1 no kernel Linux em sua versão 4.8. Também não se recomenda que se implemente um CSPRNG, mas sim que se utilize uma implementação já escrutinizada e considerada correta, pois pequenos erros de implementação podem comprometer a segurança por completo.

\section{Ferramentas de testes}

A ferramenta PractRand reinicia a coleta de amostra do gerador dobrando seu tamanho até que eventualmente o gerador fracasse, sendo 32 terabytes o tamanho máximo de uma coleta. Já o pacote TestU01 não oferece a mesma facilidade. Por ser uma biblioteca, o usuário é obrigado a escrever um programa em uma linguagem de programação conveniente para desfrutar das baterias de teste disponíveis. Por causa disso, produzimos um programa que implementa um gerador genérico, capaz de ler da entrada padrão os números aleatórios produzidos por um gerador externo ao programa. Isso efetivamente separa o gerador do testador, tornando trivial a aplicação das baterias de teste do pacote TestU01, como exemplificado por esta sessão num sistema UNIX:

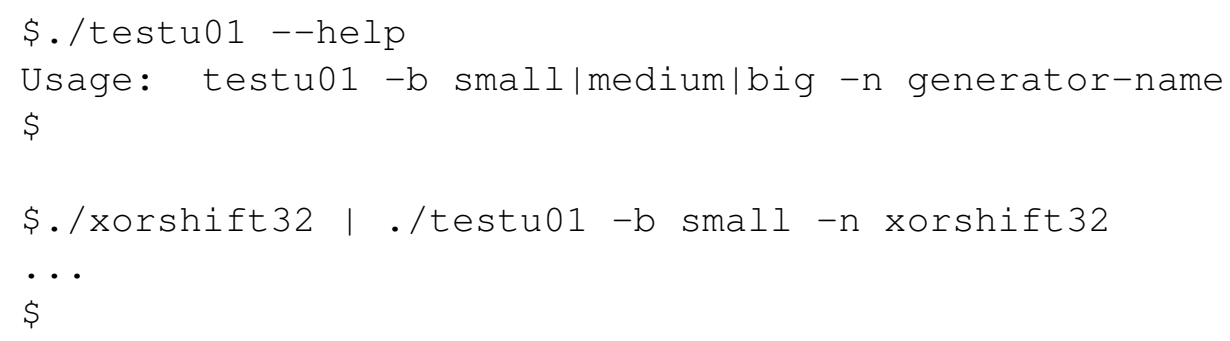


Se trocarmos a opção - b small para -b big, submetemos o gerador à bateria BigCrush do pacote TestU01, passando de uma bateria de alguns segundos para uma bateria de várias horas de duração.

Entretanto, em decorrência do design da biblioteca TestU01, não é imediatamente trivial testar, por exemplo, um gerador que produza números cujos tamanhos não sejam de 32 bits. Geradores de 64 bits, por exemplo, precisam ser adaptados porque a biblioteca testa apenas 32 bits por vez. Quando testamos o Mersenne Twister [Matsumoto and Nishimura 1998] de 64-bits, separamos os números de 64-bits em duas metades, uma contendo os bits mais significativos e na outra, os menos significativos, mas essa abordagem é penosa porque terminamos por efetuar a mesma bateria de testes 4 vezes: os bits mais significativos na ordem direta e reversa; os bits menos significativos na ordem direta e reversa. Essa alternância nos parece importante porque alguns testes prestam atenção apenas aos bits mais significativos e outros, aos menos significativos.

Outro experimento que fizemos foi submeter alguns geradores a testes específicos. Por exemplo, o Mersenne Twister, mt 19937, fracassa em questão de segundos quando submetido ao linear complexity test, coletando-se uma amostra de tamanho 50,000, embora passe sem problemas pela bateria Smal lCrush [L'Ecuyer and Simard 2007], que não inclui o linear complexity test [L'Ecuyer and Simard 2007, seção 5.1, página 17].

\section{Resultados e trabalhos futuros}

Analisamos geradores embutidos em linguagens de programação populares como Java, Python, PHP, C++ e Racket com a menor bateria de testes do TestU01. A Tabela 1 exibe a quantidade de fracassos de cada gerador, bem como enumera algumas de suas propriedades como tamanho de seu estado interno em bits e período. Na Tabela 1, o número de fracassos significa quantas estatísticas no total não passaram pelo escrutínio da bateria. Note que um mesmo teste estatístico pode gerar mais de uma estatística, como é o caso de testes como random walk [L'Ecuyer and Simard 2007, seção 5.2, página 18].

Tabela 1. Resultado de geradores populares sob escrutínio de SmallCrush, a bateria pequena da biblioteca TestU01 [L'Ecuyer and Simard 2007]

\begin{tabular}{c|c|l|c|c} 
linguagem & gerador & período & estado & fracassos \\
\hline Java & drand48 & $2^{31}$ & 48 & 5 \\
Python, PHP & mt19937 & $2^{19937}-1$ & 20032 & 0 \\
C++ & minstd & $2^{31}-2$ & 32 & 9 \\
Racket & mrg32k3a & $2^{191}-209$ & 256 & 0
\end{tabular}

Ambas linguagens Python e PHP incluem o mt19937. Apesar do sucesso na bateria SmallCrush, o mt 19937 fracassa no linear complexity test em questão de segundos, além de ter seu estado interno totalmente determinado coletando-se 624 números [O’Neill 2014, seção 2.2, página 8]. Assim, Racket, da família Lisp, é a exceção dessa amostra, pois usa o mrg32 k3a, desenvolvido pelo autor do TestU01, que passa inclusive pela bateria BigCrush [L'Ecuyer and Simard 2007, seção 7, Table I, página 27]. Esse pequeno experimento indica que a qualidade de geradores oferecidos por linguagens de programação populares não é alta. 
Como trabalhos futuros, concebemos que geradores possam ser comparados e classificados de acordo com a abordagem que discutimos na seção 6. As ferramentas que desenvolvemos e apresentamos na seção 8 podem ser incrementadas. Por exemplo, com a biblioteca TestU01, usuários são obrigados a escrever programas só para tirarem proveito de um teste específico: escrevemos um programa apenas para testar o mt 19937 contra o linear complexity test. É concebível, portanto, uma ferramenta que teste um gerador apenas contra um conjunto específico de testes. Outra limitação do pacote TestUO1 é a restrição de 32 bits no output dos geradores, dificultando o teste imediato de geradores que trabalhem com output de outros tamanhos.

\section{Referências}

Bassham III, L. E., Rukhin, A. L., Soto, J., Nechvatal, J. R., Smid, M. E., Barker, E. B., Leigh, S. D., Levenson, M., Vangel, M., Banks, D. L., et al. (2010). NIST SP 800-22 Rev. 1a. A Statistical Test Suite for Random and Pseudorandom Number Generators for Cryptographic Applications.

Bernstein, D. J. (2008). ChaCha, a variant of Salsa20. In Workshop Record of SASC, volume 8 , pages $3-5$.

Bernstein, D. J., Chang, Y.-A., Cheng, C.-M., Chou, L.-P., Heninger, N., Lange, T., and Van Someren, N. (2013). Factoring RSA keys from certified smart cards: Coppersmith in the wild. In International Conference on the Theory and Application of Cryptology and Information Security, pages 341-360. Springer.

Brown, R. (2004). DieHarder. Duke University Physics Department Durham, NC, pages 27708-0305.

Doty-Humphrey, C. (2014). PractRand: http://pracrand.sourceforge.net.

Goldberg, I. and Wagner, D. (1996). Randomness and the Netscape browser. Dr Dobb's Journal-Software Tools for the Professional Programmer, 21(1):66-71.

Heninger, N., Durumeric, Z., Wustrow, E., and Halderman, J. A. (2012). Mining Your Ps and Qs: Detection of Widespread Weak Keys in Network Devices. In USENIX Security Symposium, volume 8, page 1.

Hoare, C. A. R. (1962). Quicksort. The Computer Journal, 5(1):10-16.

Johnson, G. (2014). gjrand: http://gjrand.sourceforge.net/.

Knuth, D. E. (1997). The Art of Computer Programming, volume 2, seminumerical algorithms. Addison-Wesley Longman Publishing Co., Inc., Boston, MA, USA, 3 edition.

L'Ecuyer, P. and Simard, R. (2007). TestU01: a C library for empirical testing of random number generators. ACM Transactions on Mathematical Software (TOMS), 33(4):22.

Linux Programmer's Manual (2017). Veja “man 5 proc”.

L'Ecuyer, P. and Simard, R. (2005). TestU01: a software library in ANSI C for empirical testing of random number generators: User's guide, detailed version. Département d'Informatique et de Recherche Opérationnelle Université de Montréal.

Marsaglia, G. (1996). DIEHARD, a battery of tests for random number generators. 
Matsumoto, M. and Nishimura, T. (1998). Mersenne Twister: a 623-dimensionally equidistributed uniform pseudo-random number generator. ACM Transactions on Modeling and Computer Simulation (TOMACS), 8(1):3-30.

O’Neill, M. E. (2014). PCG: A Family of Simple Fast Space-Efficient Statistically Good Algorithms for Random Number Generation. Technical Report HMC-CS-2014-0905, Harvey Mudd College, Claremont, CA.

Pollard, J. M. (1975). A Monte Carlo method for factorization. BIT Numerical Mathematics 15 (3): 331-334.

Rivest, R. (1992). The MD5 Message-Digest Algorithm, RFC 1321.

Stinson, D. R. (2006). Cryptography, Theory and Practice. Chapman and Hall, CRC, 3 edition.

Galoá $\{$ Este trabalho foi publicado utilizando o Galoá proceedings 